\title{
A day in the life of marine sulfonates
}

\author{
Lab-based studies combined with metatranscriptomic and metabolomic field analyses reveal \\ important diel-linked roles for sulfonates in the major classes of phytoplankton producing \\ them, and in the environment, where they feed ubiquitous heterotrophic bacteria.
}

Marine microbial nutrient cycling constitutes a vast and complex metabolic network in which photoautotrophs and heterotrophs intimately interact. Phytoplankton are vital to this network, generating a plethora of organic carbon molecules to levels approaching those made by terrestrial plants $^{1}$. A significant portion of these are organosulfur molecules, representing up to $18,600 \mathrm{Tg}$ of oceanic sulfur ${ }^{2}$. Organosulfur molecules are beneficial to the organisms producing them and to the environment upon their release, stimulating chemotaxis, shaping microbial communities and supporting the carbon, sulfur and energy requirements of heterotrophs ${ }^{3}$. The sheer diversity of the molecules and organisms involved in these processes has made deciphering interactions between marine photoautotrophs and heterotrophs challenging.

Durham et al. ${ }^{4}$ attempt to untangle some of these interactions by focusing on $\mathrm{C}_{2}$ - and $\mathrm{C}_{3}$-sulfonates (compounds containing a carbon-sulfur bond ( ${\left.\mathrm{R}-\mathrm{SO}_{3}-\right)}^{-}$) (Fig.1). Sulfonates constitute up to $95 \%$ of sulfur in terrestrial soil, being key microbial nutrient sources ${ }^{5}$. Recent work suggests they are also important in Earth's oceans because diatoms can contain mM levels of e.g., 2,3-dihydroxypropane-1sulfonate (DHPS) ${ }^{6}$, roseobacters massively upregulate sulfonate transport and catabolism when cocultured with diatoms ${ }^{7}$, and the genetic potential for sulfonate catabolism is abundant in surface ocean bacterial genomes ${ }^{8}$. Durham et al. build on these findings by demonstrating that environmental sulfonate production is linked to diel cycling in the major classes of phytoplankton, at levels that stimulate its import and catabolism by Earth's most abundant heterotrophs.

An impressive selection of phytoplankton and heterotrophic bacteria were screened for $\mathrm{C}_{2}-$ and $\mathrm{C}_{3}-$ sulfonates by targeted metabolomics (Fig.1). Sulfonates were either undetected, e.g., DHPS and isethionate, or present at low levels (typically $<1 \mu \mathrm{M}$ ) in heterotrophic bacteria. In phytoplankon they were detected at higher levels in taxon-specific combinations; $\mathrm{C}_{2}$-sulfonates were widespread across the phytoplankton, whereas $\mathrm{C}_{3}$-sulfonates were more pronounced in diatoms and haptophytes, with DHPS reaching $\mathrm{mM}$ levels in most. This strongly supports globally abundant phytoplankton, not just diatoms, as the most likely source of marine sulfonates that feed diverse heterotrophs. Given the taxon-specific production of these sulfonates, they may be important in recruiting specific heterotrophs.

There are few mechanistic studies of phytoplankton sulfonate production ${ }^{9}$, and sulfonate synthetic pathways in these organisms are uncertain. The authors propose phytoplankton sulfonate metabolic pathways based on the presence of homologues to ratified sulfur metabolic genes (Fig.1).

Metabolism of cysteine and serine to taurine and subsequently to isethionate was predicted in most phytoplankton (Fig.1). Interestingly, phytoplankton lacked the complete sulfoquinovose catabolic pathway, a ratified pathway for DHPS production ${ }^{10}$. Durham et al. propose well-reasoned alternative DHPS synthesis pathways, including the conversion of cysteate through sulfolactate to DHPS, or from cysteinolic acid ${ }^{9}$. These predicted pathways and candidate genes are plausible, but confirmation involving labelled substrates and/or enzyme characterisation is still required. This is important given 
the conclusions stemming from the regulation of these genes in the environment, and for future studies using them as reporters of environmental sulfonate synthesis.

Metabolomics and metatranscriptomics were used effectively on North Pacific samples to provide evidence that phytoplankton sulfate assimilation and sulfonate synthesis are coupled to diel rhythm, particularly in diatoms and haptophytes. Apart from the five identified sulfonates, 19 unknown sulfur-containing molecules were also detected, highlighting the need for studies on novel organosulfur molecules ${ }^{11}$. Alongside the diel-regulated sulfonates detected (isethionate, DHPS, taurine and likely, cysteinolic acid), several sulfate assimilation and candidate sulfonate synthesis genes (e.g., $C D O 1, S D H$ and $C O A$ ) displayed diel periodicity in diatoms and/or haptophytes. It will be interesting to see these findings confirmed in future pure culture studies. Completing the cycle, bacterial transcripts of ratified sulfonate catabolic genes ${ }^{8,12,13}$ were relatively abundant in bacterial metatranscriptomes from samples collected at dawn. Environmentally abundant SAR11, SAR116, Roseobacter and Gammaproteobacteria lineages (Fig.1) were most active, and their sulfonate catabolic pathways were inferred for taurine, isethionate and/or DHPS, theoretically generating pyruvate, acetyl CoA and/or bisulfite for assimilation. Furthermore, the authors isolated a North Pacific SAR11 strain and show that taurine and DHPS are effective carbon and energy sources, providing further evidence for the importance of phytoplankton-derived sulfonates in oceanic heterotroph productivity.

Whilst sulfonates accumulate to mM levels in some phytoplankton, we are left wondering why. Indeed, the same is true for other organosulfur molecules, e.g., dimethylsulfoniopropionate (DMSP), which can accumulate to far higher intracellular concentrations in phytoplankton, and has been the focus of numerous studies ${ }^{14}$. Like DMSP, some sulfonates are proposed to function as osmolytes ${ }^{15}$, and Durham et al. show that DHPS accumulation by Thalassiosira pseudonana is regulated by salinity, alongside osmolytes including DMSP and proline. It would be interesting to measure whether transcription of the proposed candidate sulfonate synthesis genes is regulated in the same way. Durham et al. also posit that sulfonates may maintain redox balance during phototrophic metabolism. An important focus of future work should therefore be the ratification of candidate sulfonate synthesis genes and their mutation in model phytoplankton, to establish their physiological effects. As many similar molecules accumulate to comparable or higher levels than sulfonates in e.g., diatoms, it may be difficult to discern a phenotype or role. Additionally, the localisation of sulfonates and their synthetic enzymes within phytoplankton may help to infer their function as e.g., organelle-specific osmolytes ${ }^{16}$.

Finally, how do sulfonates end up in the oceans - through active export by phytoplankton, or from cell lysis or leakage? Do heterotrophs provide phytoplankton nutrients in return for their sulfonate treats - a similar situation to that described for some hormones/vitamins ${ }^{7,17}$ ? Do specific bacteria demonstrate chemotaxis towards sulfonates? This study has opened a window into these marine networks, and in doing so provided plenty of avenues for future research. 
Figure:
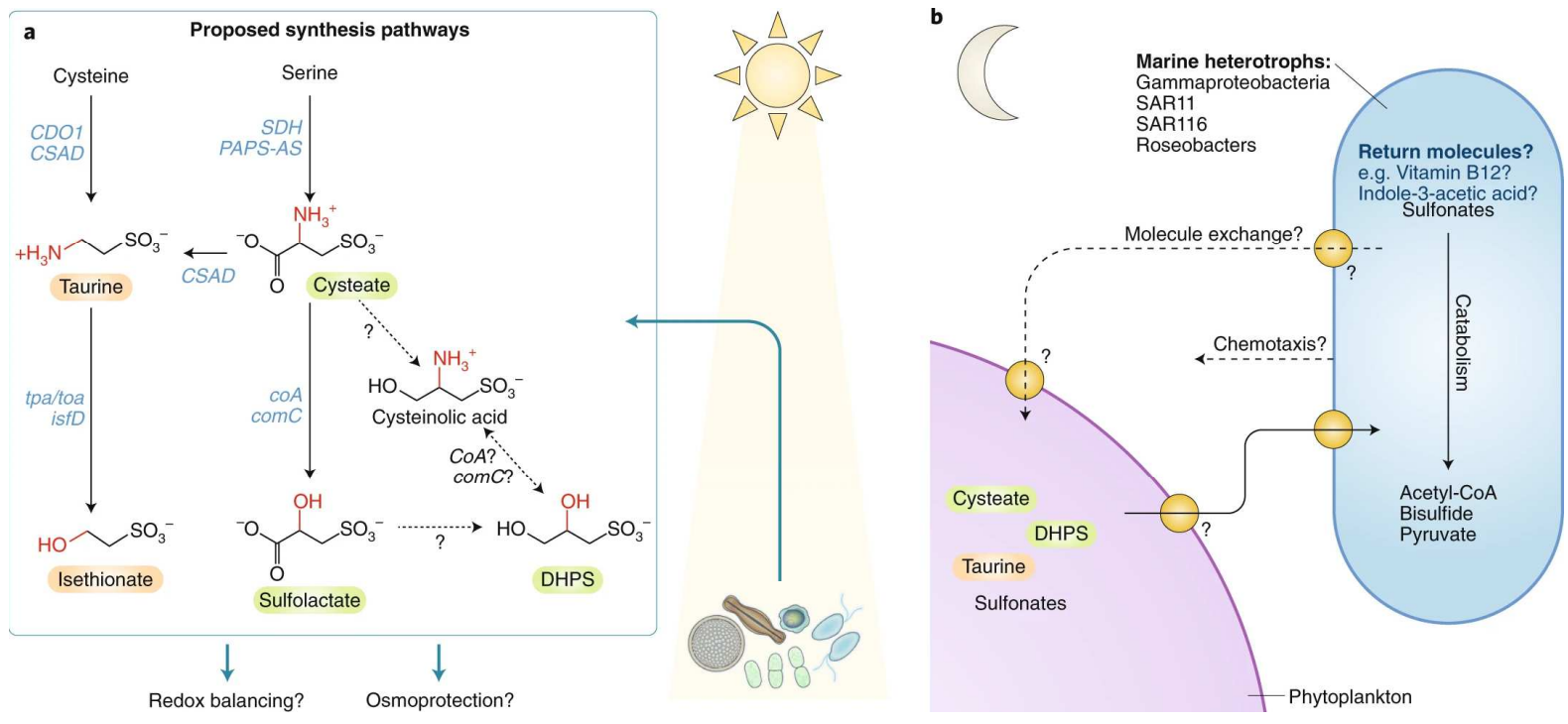

The proposed sulfonate metabolic network showing the diel cycle-linked production and release of sulfonates by many phytoplankton, and uptake by diverse heterotrophic bacteria.

a, Accumulation of five sulfonates (taurine, isethionate, DHPS, cysteate and sulfolactate) in phytoplankton during the daytime suggests that they may function in redox balancing. Sulfonates may also act as osmoprotectants. Phytoplankton sulfonate synthesis pathways were theorised on the presence of homologues to ratified sulfonate biosynthesis genes. Isethionate is derived from taurine through a taurine aminotransferase (encoded by $t p a / t o a$ ) and sulfoacetaldehyde reductase (isfD), genes for which were detected in most phytoplankton (diatoms did not possess isfD). Taurine can be derived from cysteine through oxidation and decarboxylation steps, inferred by genes encoding putative cysteine dioxygenases (CDO1) and cysteine sulfinic acid decarboxylases (CSAD). Cysteate can be produced from serine through sulfonation reactions (SDH and PAPA-AS encoding serine dehydratase and 3'-phosphoadenylyl sulfate:2-aminoacrylate C-sulfotransferase). Cysteate can be converted to taurine through decarboxylation via CSAD, or to sulfolactate via genes encoding cysteate aminotransferase ( $C O A)$ and sulfolactate dehydrogenase (comC). Hypothetical DHPS synthesis pathways from cysteate via cysteinolic acid, or via sulfolactate, were proposed without direct knowledge of the genes or enzymes involved. $\mathbf{b}$, At night, a portion of the sulfonates are released from phytoplankton (by unconfirmed processes, e.g., transport, lysis or leakage) and are imported by marine heterotrophic bacteria for catabolism to produce e.g., acetyl-CoA, bisulfide and pyruvate, for carbon, energy and sulfur requirements. Questions arising on the genes involved in DHPS production, mutualistic exchange of molecules and chemotaxis are indicated by a question mark (?); $\mathrm{C}_{3}$-sulfonates are circled in green; $\mathrm{C}_{2}$-sulfonates are circled in orange; purely hypothetical reactions are indicated by dotted lines. 


\section{References:}

1. Field, C. B. Science 281, 237-240 (1998).

2. Ksionzek, K. B. et al. Science 354, 456-459 (2016).

3. Levine, N. M. Science 354, 418-419 (2016).

4. Durham, B.P. et al. Nature Microbiol, https://doi.org/10.1038/s41564-019-0507-5 (2019).

5. Kertesz, M.A. FEMS Microbiol Revs, 24, 135-175 (2000).

6. Durham, B.P. et al. PNAS, 112, 453-457 (2015).

7. Amin, S.A. et al. Nature, 522, 98-101 (2015).

8. Landa, M. et al. ISME J, https://doi.org/10.1038/s41396-019-0455-3 (2019).

9. Busby, W. F. \& Benson, A. A. Plant Cell Physiol, 14, 1123-1132 (1973).

10. Denger, K. et al. Nature, 507, 114-117 (2014).

11. Thume, K. et al. Nature, 563, 412-415 (2018).

12. Cook, A.M., Denger, K. \& Smits, T.H. Arch Microbiol, 185, 83-90 (2006).

13. Cook, A.M. \& Denger, K. Arch Microbiol, 179, (2002).

14. Zhang, X.H. Sci China Life Sci, https://doi.org/10.1007/s11427-018-9524-y (2019).

15. Götz, F. et al. MicrobiologyOpen 7, e00586 (2018).

16. Curson, A.R.J. et al. Nat Microbiol, 3, 430-439 (2018).

17. Kazamia, E., et al. Environ Microbiol, 14, 1466-1476 (2012). 